\title{
The Effects of Attractiveness, Dominance, and Attribute Differences on Information Acquisition in Multiattribute Binary Choice
}

\author{
ULF BoCKENHOLT \\ University of Illinois
}

\section{Dietrich Albert and Michael Aschenbrenner}

University of Heidelberg

\author{
AND \\ Franz SChMalhofer \\ German Research Center for Artificial Intelligence, Kaiserslautern
}

\begin{abstract}
Two process tracing studies investigated how the information acquisition process in a binary choice task is influenced by the overall level of attractiveness of alternatives, by the magnitude of differences in attractiveness of alternatives, and by the dominance of one alternative. All three factors influenced the subjects' information selection process regarding the multiattribute choice alternatives. Subjects selected more information when the attractiveness difference was small and when one of the alternatives was not dominant. Moreover, they considered more information when the choice alternatives were both unattractive. These findings were obtained when information was presented about the alternatives both numerically and nonnumerically. The experimental results were explained within a sequential sampling strategy framework. 1991 Academic Press, Inc.
\end{abstract}

Process tracing methods are useful for determining important factors that influence the utilization of information in a choice situation. One well-established finding of process tracing studies is that an increase in the number of choice alternatives leads to a more noncompensatory integration of information about the alternatives (Onken, Hastie, \& Revelle,

We are grateful to Jerome Busemeyer, Douglas Wedell, and Elke Weber for valuable comments on an earlier version of this manuscript. Maria Bannert, Nancy Linn, and Thomas Schneyer helped in conducting the experiments. In addition, we thank the referees for their helpful suggestions. This research was supported by the Deutsche Forschungsgesellschaft and by a grant from the Research Board of the University of Illinois. Requests for reprints should be addressed to Dr. Ulf Bockenholt, Department of Psychology, University of Illinois, 603 East Daniel Street, Champaign, IL 61820. 
1985; Svenson, 1979). The common explanation of this finding is that decision makers attempt to simplify the decision problem without making a poor choice (Payne, 1976; Wright, 1974). According to this explanation, individuals perform a cost-benefit comparison of information selection strategies and employ strategies which yield an accurate choice but involve low costs of processing information in terms of both effort and time. The simulation studies and experimental results obtained by Payne, Bettman, and Johnson (1988) provide strong support for this viewpoint.

Relatively few process tracing studies examined information acquisition behavior in a binary choice situation (Busemeyer, 1985; Montgomery, 1977; Russo \& Dosher, 1983). In particular, characteristics of information search patterns in a binary choice task, such as the process of selecting attributes as a function of different context variables, have not received much research emphasis. This may be a useful enterprise, however, as demonstrated by Schmalhofer, Albert, Aschenbrenner, and Gertzen (1986). These authors indirectly demonstrated the adaptive behavior of decision makers when choosing between two alternatives. For example, based on a post hoc analysis of attractiveness differences between alternatives, it was found that subjects considered more information about the alternatives when the overall attractiveness difference was small rather than large.

Schmalhofer et al. (1986) explained the information acquisition behavior of the subjects in terms of the criterion-dependent choice (CDC) model (Aschenbrenner, Albert, \& Schmalhofer, 1986; Albert, Aschenbrenner, \& Schmalhofer, 1989). Similar versions of this model have been presented by Busemeyer $(1982,1985)$ for one-dimensional risky choice situations and by Wallsten and Barton (1982) for probabilistic inference tasks. The CDC model posits that decision makers process information about the alternatives by comparing the alternatives with respect to the subjective evaluations of the features, one attribute at a time. It is assumed that features of the alternatives are processed only once and that they are not reconsidered after they have been selected. The results of the attributewise comparisons are accumulated over the processed attributes. When a person has accumulated enough evidence to be convinced that one alternative is better, the comparison process stops and an alternative is chosen.

More formally, let $v\left(x_{i}\right)$ and $v\left(y_{i}\right)$ denote the subjective evaluations of alternatives $x$ and $y$, respectively, on attribute $i$. The difference of the subjective evaluations, $\left\{v\left(x_{i}\right)-v\left(y_{i}\right)\right\}$, is added over the selected attributes until the absolute sum of the aggregated differences is equal to or exceeds a critical value, $k$,

$$
\mid \Sigma\left(v\left(x_{i}-v\left(y_{i}\right)\right) \mid \geqslant k .\right.
$$


The critical value, $k$, is determined by the decision maker. For a given pair of alternatives a large $k$ value implies that most or all of the attributes that characterize the alternatives have to be considered. In contrast, a small $k$ value may lead to a choice after the consideration of only a few attributes. The CDC model includes many choice strategies as special cases. For example, the CDC model with a low critical value mimics the lexicographic decision rule (Tversky, 1969). In contrast, the CDC model with a large evidence criterion becomes the additive rule (Svenson, 1979). Similarly, the majority of confirming dimensions (MCD) rule (Russo \& Dosher, 1983) is a special variant of the CDC model if the subjective value differences are coded in a binary way, so that only the direction of a difference but not its size is taken into account (see also Aschenbrenner et al., 1986).

Moreover, the size of the critical value reflects the cffort-quality tradeoff intended by the decision maker. Frequently, some of the available information about the choice alternatives may be ignored without necessarily leading to a poor choice. Consequently, the CDC model is consistent with a cost-benefit framework. The critical $k$ value may be specified in such a way that a balance is reached between the effort involved in a decision process and the quality of the choice outcome. For example, only when the selected information about two alternatives is of similar attractiveness is it beneficial to consider more information for distinguishing between them. Less information is required to select the better alternative if both alternatives differ greatly in their features' attractiveness.

The goal of the following studies was to investigate whether the CDC model is sufficiently general to describe subjects' choice behavior over different situations. We examined how individuals modify their information acquisition behavior as a function of several contextual factors. In particular, we investigated the CDC model's ability to predict the depth of the information search of a decision maker. For example, the model predicts that subjects consider more information when the attractiveness differences between two alternatives are small rather than large in order to accumulate sufficient evidence for making a choice. We constructed alternatives which varied in their overall attractiveness and then paired them (a) to obtain various overall attractiveness differences, and (b) to have dominant and nondominant relations between alternatives.

We manipulated the overall attractiveness level of the choice alternative pairs to examine if individuals consider this information when making a choice. Although the CDC model does not predict any effects of this factor, there is some indirect evidence that attractiveness of an alternative may influence information acquisition in risky choice situations (Busemeyer, 1985). We therefore manipulated this factor explicitly to examine its relevance in a multiattribute choice situation. The attractiveness dif- 
ference factor was introduced to examine the findings at Schmalhofer $e t$ al. (1986) in more detail whereas dominance was included because it has been identified in past research as one of the critical factors affecting choice behavior (Batsell \& Polking, 1986). According to the CDC model, an individual may select fewer attributes of the choice alternatives when comparing a dominant to a nondominant alternative than when comparing two nondominant alternatives because in the former case fewer attribute differences may be needed for reaching the critical evidence criterion.

\section{GENERAL PROCEDURE}

One problem in studying information search behavior is that subjects may value attributes differently. Thus, an alternative defined by a set of features may have a different value structure for different subjects. Consequently, differences in subjects' choice behavior among identical choice alternatives may be a result of either the value representation of the alternatives, the employed strategy, or a combination of both factors. The present experiments attempted to circumvent this ambiguity by equating the value structure of an alternative for all subjects. One particular value structure of an alternative may be, for instance, that it has highly attractive features on all attributes. In the present studies, subjects would then be presented with their most preferred features for all attributes selected. While the value structure is the same, or at least similar, for all subjects, the presented features for each alternative may vary among subjects because subjects may not prefer the same features of an alternative. An additional advantage of presenting alternatives with a known value structure is that we can considerably reduce variation resulting from individual differences in valuing the attributes.

Of course, equating the value structure for the subjects requires knowledge about the evaluation of the alternatives' features. We selected two different approaches to this problem. In the first experiment, we presented alternatives which were characterized by numerical attractiveness ratings on a set of attributes and asked subjects to interpret these attractiveness ratings as if they were their own subjective assessments of the alternative's features. In the second experiment, alternatives were characterized by their features on a set of attributes. Before the choice part of the experiment subjects were asked to rank-order the alternative's features according to their attractiveness. These rank-orders were then used to construct alternatives with a certain value structure. The applicability of the CDC model to these two quite different presentation formats demonstrates the generality of this approach to modeling information acquisition. 


\section{EXPERIMENT I}

Choice pairs were shown to each subject on a video screen. The alternative's features were represented in the form of numerical attractiveness ratings. We used numerical ratings because they simplified the task of constructing the alternatives' value structures. Moreover, in contrast to most semantic stimuli, the meaning and value of numbers are simple and relatively uniform between subjects (Huber, 1980; Wallsten, Budescu, Rapoport, Zwick, \& Forsyth, 1986). While the video screen always revealed the names of the attributes on which the alternatives were evaluated, the features of a choice pair had to be separately uncovered for each attribute. The inspected feature pairs as well as the subjects' choices were recorded for every choice pair.

\section{Method}

\section{Subjects}

Sixteen students from the University of Heidelberg participated in the experiment. These students were volunteers who responded to a notice in a student cafeteria. Each subject was tested individually and was paid ten marks (about \$5) per hour.

\section{Apparatus}

The experiment was computer controlled; 10 attributes and the corresponding 20 ratings of a choice pair were displayed on a video terminal driven by an Apple II microcomputer. Using a joystick, subjects could move to an attribute name and by pressing a button could read the corresponding numerical ratings of the choice pair for the selected attribute. When selecting the next attribute name, the numerical ratings of the previous attribute were erased, and the ratings of the selected attribute were presented. The ratings were erased to ensure that the number of selected attributes was not influenced by a rereading of the information on the screen.

\section{Design and Procedure}

The choice task consisted of choosing a summer vacation location in Europe. Each subject was given 84 trials in which she or he had to choose between vacation locations. The features' evaluations of the choice alternatives were presented as ratings on an attractiveness scale ranging from 1 (very unattractive) to 7 (very attractive). The subjects were instructed to consider these ratings as their own evaluations of the alternatives' features. In order to get acquainted with the rating scale, subjects rated some 
TABLE 1

Sample Choice Pair in Experiment I

\begin{tabular}{lll}
\hline \multicolumn{1}{c}{ Vacation location } & A & B \\
\hline Rainy days & 5 & 5 \\
Temperature & 2 & 7 \\
Leisure activities & 6 & 5 \\
Purchasing power (German Mark) & 7 & 6 \\
Landscape & 4 & 7 \\
Location & 5 & 4 \\
Historical sites & 6 & 6 \\
Floral environment & 4 & 5 \\
Popularity & 3 & 7 \\
German spoken & 7 & 7 \\
\hline
\end{tabular}

Note. The presentation order of the values is fixed. The order of the attribute labels is determined by the subject and may therefore differ from subject to subject.

alternatives' features on the same attractiveness scale with unrelated material. A sample choice pair is presented in Table 1 .

A $2 \times 3 \times 2$ factorial design with the factors attractiveness difference, dominance, and attractiveness of alternatives was used to construct the value structure of the choice pairs. For each cell, seven pairs of alternatives were generated, thus yielding 84 pairs of alternatives. The value structure was constructed according to the rules outlined below.

Attractiveness differences of absolute differences. Given a seven-point rating scale, differences between alternatives' features ranged from -6 to +6 (i.e., 1-7 to 7-1). The absolute difference values 4,5 , and 6 were coded as large differences and the remaining differences $0,1,2$, and 3 as small differences. In the high difference condition, $80 \%$ of the absolute differences between the alternatives' features were large, while in the low difference condition, only $20 \%$ of the absolute differences were large. This manipulation generated choice alternatives with a mean difference of 3.4 and 1.4 in the high and low difference conditions, respectively.

Dominance or signed differences. In addition to manipulating the size of the differences, we varied the direction of the attribute differences. This manipulation yielded dominant and nondominant relations between the alternatives. An alternative is dominant if its features are at least as good as the features of the second alternative on all attributes. In the dominant conditions, the alternative's ratings presented on the left (right) side of the video screen were equal or superior to the alternative on the right (left) side of the screen. In the nondominant condition, neither of the two alternatives was consistently superior. The dominant and nondominant conditions were operationalized by drawing rating differences from a uniform distribution either with the value range ( 0 to 6 ; i.e., the alter- 
native positioned at the left part of the screen was superior) or with the range ( -6 to 0 ; i.e., the right alternative was superior), while for the nondominant level, differences were drawn from the full value range $(-6$ to 6).

Attractiveness of alternatives. The same difference, 2 , can be calculated from the rating scale values 7 and 5, for example, or from the values 3 and 1 . In the first case, the values of both alternatives' features are more attractive than in the second case. These two representations of differences constituted the third factor, called attractiveness, with the two levels, high and low. The differences obtained from the factorial combination of the above two factors were mapped into low and high attractive rating values. Because for large differences $(4,5$, and 6$)$ the mapping range into high or low attractiveness ratings was very limited, the potential effect of this manipulation was expected to be stronger for small rather than large difference values. Overall, the mean attractiveness of the choice alternatives was 4.8 and 3.2 in the high and low attractiveness conditions, respectively.

It is important to note that, regardless of the attribute selected, the numerical ratings displayed on the screen were the same for every subject. For example, in the sample choice trial in Table 1 the ratings 5 and 5 (of the first and second alternative, respectively) were presented for the first selected attribute regardless of the selected attribute's label. For the second selected attribute the value pair 2 and 7 was presented regardless of the selected attribute's label, etc.

Subjects were given 84 choice trials. At the beginning of every choice, subjects saw only the names of the 10 attributes which described the alternatives. They could move a pointer to an attribute, and by pressing a button subjects could inspect the corresponding attractiveness ratings of the choice pair. After examining these ratings, subjects could request the ratings of another attribute. This procedure was repeated until a subject wanted to make a choice. Subjects were instructed to inspect as many rating pairs or attributes (up to 10) as they liked before choosing an alternative. In addition to the choices, the selected attributes were recorded and the number of processed attributes were calculated for every choice pair.

\section{Results}

An analysis of the number of processed attributes revealed that subjects employed selective information processing rather than processing all features of a choice pair. From the 10 possible attributes of an alternative pair, subjects inspected only five attributes on the average. Due to the large number of choices, we examined further the distributions of the number of attributes selected which are displayed in Fig. 1. The 


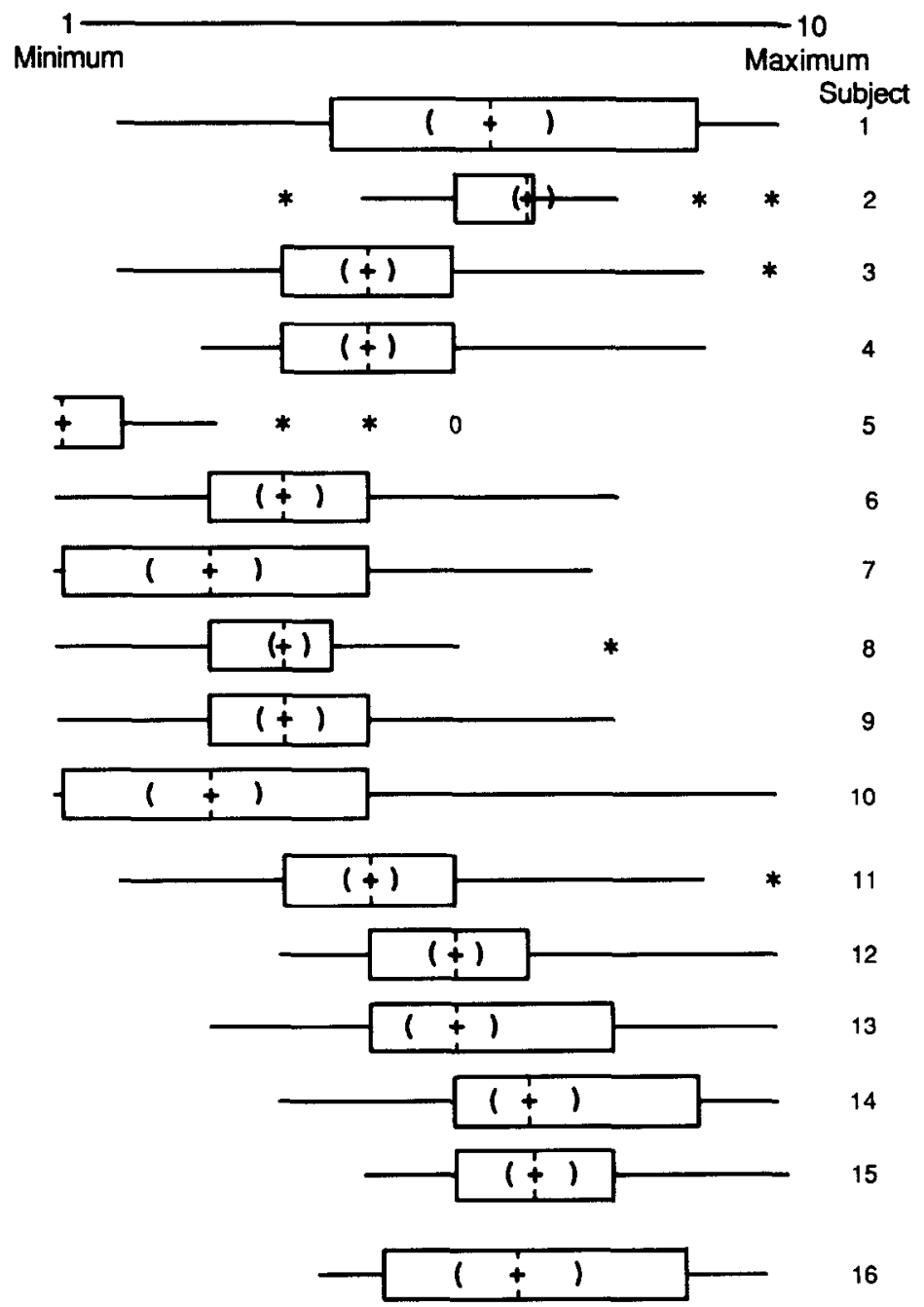

Fig. 1. Boxplots of the 16 subjects in Experiment I. The median number of selected attributes is marked with a + , and the left and right edges of the box surrounding the median correspond to the 25 th and 75 th percentile, respectively. Outside values are marked with an * and far outside values are marked with a 0 . The parentheses around the medians define the subjects' simultaneous $\mathbf{9 5 \%}$ confidence intervals for the medians.

nonnormality of the distributions is readily apparent. For all but two subjects, the distribution is negatively skewed, demonstrating that subjects more frequently consider a large rather than a small number of attributes. Subjects did not inspect the same number of attributes for every choice pair; instead, they varied considerably in inspecting at- 
tributes. Subjects' median numbers of selected attributes ranged from 1 to 7 .

We first averaged the number of selected attributes of the seven pairs of alternatives generated in each condition and then, using a repeated measures ANOVA model, we analyzed the averaged number of selected attributes at the subject and group level. Because the results obtained from the group data are representative of the analysis on the individual level, we restrict the following presentation of results to the collective data for all subjects. The design encompassed the main effects of the three factors, overall attractiveness, attractiveness differences, and dominance, as well as their corresponding interactions. Except for the difference $\times$ attractiveness interaction $(F(1,15)=5.71, p<.03)$, no interactions were significant. However, the three main effects were significant $(F(1,15)=37.38, p<.001 ; F(1,15)=17.71, p<.001 ; F(2,14)=4.83, p$ $<.05$ for the difference, attractiveness, and dominance factors, respectively) (Similar results were obtained when using an ordinal regression model for the analysis of the selected attribute data). Figures 2 and 3 depict the dominance $\times$ difference and the difference $\times$ attractiveness effects, respectively.

The expected difference effect is strong; subjects selected more attributes for small differences between features and not for large feature differences. An effect similar to the difference factor but smaller in influence can be observed for the dominance factor. Subjects terminated their

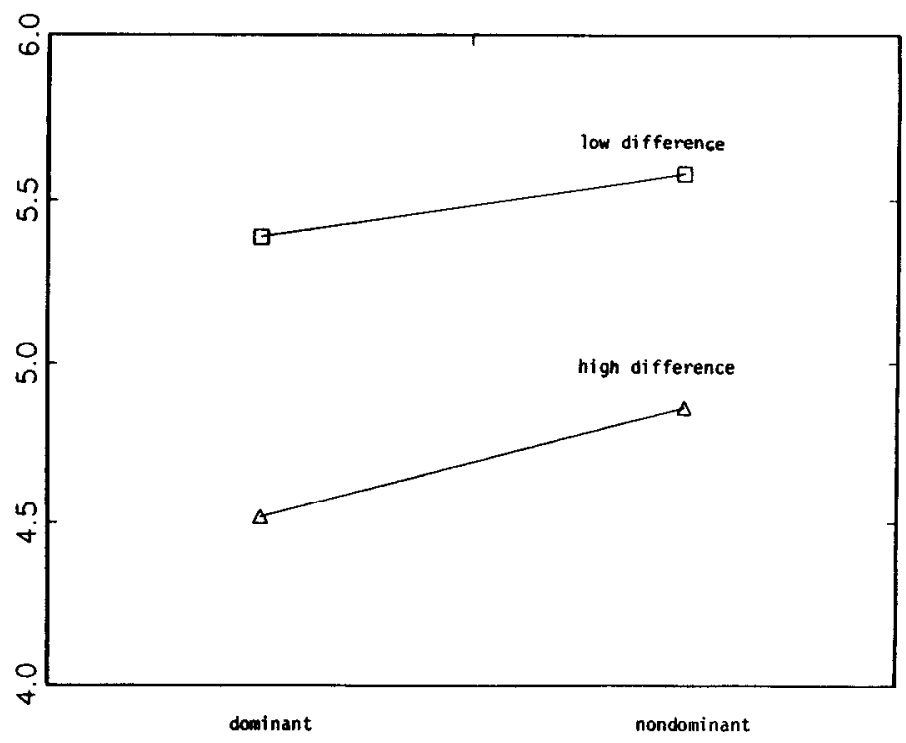

FIG. 2. The number of selected attributes in the dominance and difference conditions. 


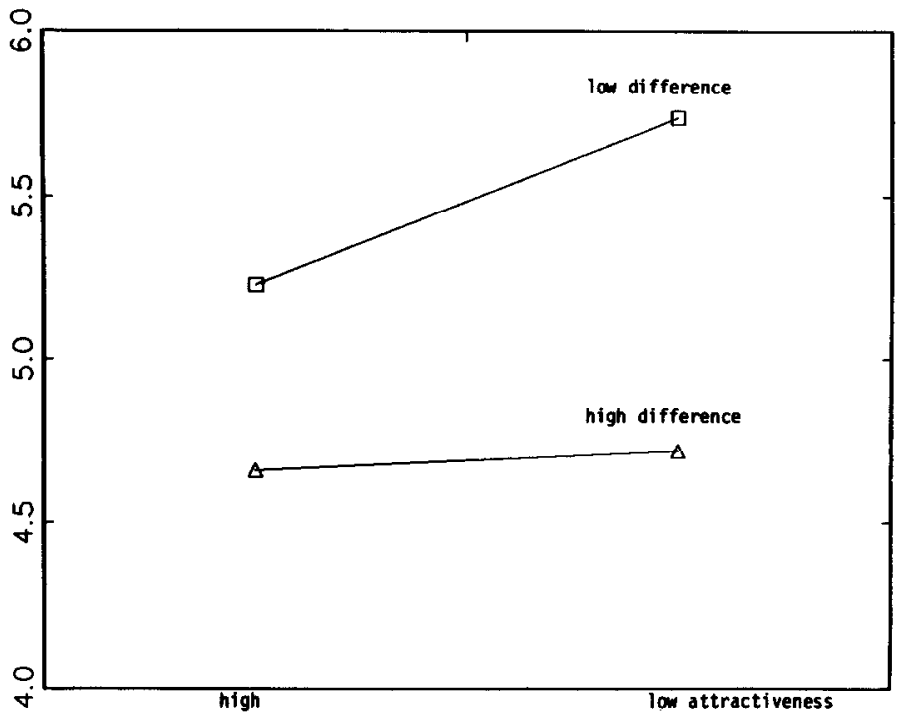

FIG. 3. The number of selected attributes in the attractiveness and difference conditions.

information searches earlier when an alternative was dominant than when none of the alternatives were dominant. A planned comparison contrasting the two conditions of a dominant alternative displayed at the left or right panel of the screen yielded no significant difference $(F(1,15)=0.24$, $\mathrm{p}>\mathbf{0 . 1}$ ). Thus, we did not obtain a position effect.

Finally, the number of selected attributes was strongly influenced by the overall attractiveness of the alternatives. All subjects but one exhibited a pattern of considering more attributes when the attractiveness values were low rather than high. However, the effect size of the attractiveness factor in the high difference condition was considerably weaker than in the low difference condition which led to a significant difference $\times$ attractiveness interaction. A partial interaction analysis yielded a significant effect due to the attractiveness factor under the small difference condition $(F(1,15)=13.59, p<.002)$ but no effect under the large difference condition $(F(1,15)=0.91, p>.10)$. This result is probably due to the fact that there were fewer opportunities to manipulate the attractiveness values in the high difference condition than in the low difference condition.

\section{Discussion}

Subjects did not select the same amount of information about each choice pair. Instead, we found systematic and strong effects of the manipulated value structure of the alternatives. Strategies which require complete information processing of the attribute information cannot ac- 
count for these results. The difference effect obtained in the present study corroborates results obtained by Schmalhofer et al. (1986). They found that subjects process more attributes for similar choice pairs in comparison to dissimilar choice pairs. Their result was obtained using a post hoc analysis of a choice experiment with described features. In contrast, we systematically varied the differences between alternatives to investigate the phenomenon. The effect of the dominance factor is related to the effect of the difference factor. The significance of both factors indicates that subjects used, at least partially, an attributewise information processing strategy because dominance can only be recognized when a comparison of the numerical ratings is performed for every attribute (Russo \& Dosher, 1983).

Both results provide support for the CDC model. In order to obtain the same critical difference for making a choice, less information is needed when one alternative is dominant than when neither of the alternatives is dominant. Moreover, subjects took the size of the difference into account in the two conditions with one dominant alternative and either small feature differences or large feature differences. This result cannot be accounted for by two special cases of the CDC model, the MCD rule and the lexicographic rule. Neither strategy is sensitive to the size of an attribute difference. Instead, they predict that the same number of attributes is selected under either condition.

While these findings support the assumption that subjects process information attributewise, the effect of the attractiveness factor suggests that subjects take into account more than just the differences in attractiveness. Specifically, the perceived overall level of attractiveness of the alternatives' features influences the number of attributes selected. Initially, the effect of the attractiveness factor seems incompatible with the CDC model. However, there are several ways to reconcile the two. One explanation is that subjects did not perceive the numerical rating scale values as equally spaced. Subjects could code the same small difference between two attractive alternatives as larger than between two unattractive alternatives. Although this explanation accounts for the observed attractiveness effect, it would be unusual that all subjects but one would interpret the spacing of the numerical rating scale values in the same fashion and process more attributes for unattractive than for attractive rating values. Moreover, it seems likely that an unequal spacing of the rating scale values would affect the choice proportions for high and low attractive alternatives. This prediction, however, was disconfirmed by a $\chi^{2}$ test of the equality of choice probabilities in the two attractiveness conditions which yielded a nonsignificant result $\left(\chi^{2}=0.36, d f=1\right)$. Finally, indirect evidence against this hypothesis can be obtained from research about the impact of negativity weighting in choice situations (e.g., Birnbaum, 1974; Levin, 1975; Lynch, 1979). A common finding of 
this research is that pieces of negative information are weighted more highly than positive information in an overall comparison of two choice alternatives.

An alternative explanation of the results is that the setting of the critical value may be influenced by the attractiveness of the alternatives. Thus, the critical value may be higher in a comparison of two unattractive alternatives than in a comparison of two attractive alternatives. It may be suggested that subjects are more likely to be satisfied with either choice outcome when choosing between two attractive options. This may lead them to adopt a low critical value and, consequently, to terminate their selection of attributes early. In contrast, when choosing between two unattractive alternatives, subjects may employ a higher critical value and consider more attributes to ensure that the chosen alternative is indeed the "better" onc. This interpretation of the data is supported by the viewpoint that a subject's choice behavior is guided by cost-benefit considerations.

In order to test this explanation we fit a version of the CDC model to predict the choices and the number of attributes selected by the subjects. Assuming that the numerical ratings displayed on the screen are identical to the subjective evaluations, we added the rating differences until they were equal to or exceeded some cutoff value. The number of selected attributes for all 84 choice trials was then recorded and compared to the observed number by means of the association coefficient $e_{P O}$,

$$
e_{P O}=2 S_{P O} /\left\{S_{P}^{2}+S_{O}^{2}+\left(M_{P}-M_{O}\right)^{2}\right\}
$$

where $P$ is the predicted number of selected attributes and $O$ is the observed number of selected attributes. $S_{P O}$ refers to the covariance, and $S_{P}{ }^{2}, S_{O}{ }^{2}, M_{P}$, and $M_{O}$ are variances and means of the predicted and observed number of attributes, respectively. This association coefficient suggested by Zegers (1986) is a chance-corrected coefficient that describes the relationship between two variables measured on an absolute scale. It measures the similarity between the observed and predicted number of attributes by taking into account both the dispersions and the differences between both variables. As a result, this coefficient is smaller in absolute value than the Pearson correlation coefficient. More details about this coefficient can be found in Stine (1989).

The above procedure was repeated for all possible critical values until a critical value was found which maximized $e_{P O}$. This version of the CDC model is the most parsimonious because it requires the determination of only one parameter, the critical value $k$. In order to test the performance of the CDC model we determined the sampling distribution of $e_{P O}$ under the condition that there is not relationship between the number of sampled attributes and the value structure of the alternatives. We generated 
each element of the sampling distribution by drawing 84 values between 1 and 10 from a uniform distribution and by computing the optimal $e_{P O}$ value for this "data set." A histogram of the generated distribution based on $100 e_{p o}$ 's is displayed in Fig. 4. The 95th percentile corresponds to a value of .21 and the 99th percentile to a value of .25 (similar results were obtained using a bootstrap procedure by randomly drawing (with replacement) from the subjects' distribution of selected attributes).

When fitting the data of the subjects, we obtained for 13 subjects association values larger than the 99 th percentile of .25 , for one subject a value above .21 , and for two subjects values below .21 . The mean association value obtained from all subjects is .35 . (In comparison, the average Pearson correlation coefficient between the observed and predicted number of attributes is .45 . However, this value underestimates the correlation coefficient obtained when using it in the estimation procedure.) Obviously, for the majority of the subjects this simple version of a CDC model predicts the observed number of selected attributes much better than would be expected by chance.

The fitted version of the CDC model predicts about $88 \%$ of the subjects' choices correctly; $71 \%$ is the lowest percentage of correctly predicted choices and for eight subjects the choice predictions are higher than $90 \%$. In comparison, the correct number of predictions is .90 if the observed number of attributes is used. This difference between both predictions is significant according to the Wilcoxon test $(p<.05)$. The additive rule based on the obviously incorrect assumption that subjects considered all the presented information predicts $86 \%$ of the choices correctly. This prediction is significantly worse than the CDC model prediction (Wilcoxon test, $p<.01$ ).

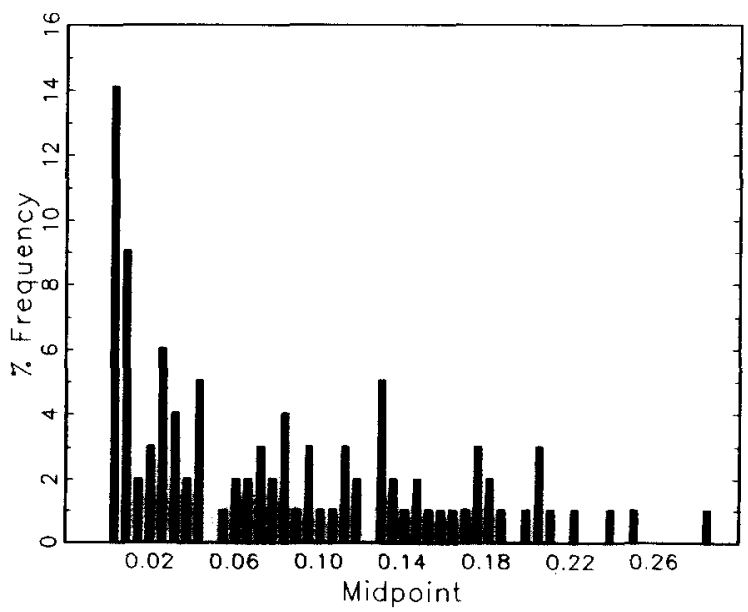

Fig. 4. Histogram of the sampling distribution of the association coefficient $e_{P O}$. 
The attractiveness effect was modeled by splitting the number of selected attributes into halves, the first half corresponding to the attractive alternatives and the remaining half corresponding to the less attractive alternatives. For each half we estimated that optimal critical value. Not surprisingly, the $e_{P O}$ values increased substantially. A comparison between the generated sampling distribution for the CDC model with two critical values and the estimated $e_{P O}$ 's obtained from the subjects' data indicated that all but one subject had a fit statistic higher than the 95th percentile of the sampling distribution with 14 subjects having a fit statistic higher than the 99th percentile. A Wilcoxon test comparing both critical values over subjects showed that a higher critical value was estimated for low rather than for high attractive alternatives $(p<.05)$. Moreover, the mean percentage of correctly predicted choices increased slightly from 88 to $89 \%$.

We further tested the adequacy of the fitted version of the CDC model by examining whether the predicted number of selected attributes capture the design effects observed in the data. Using a repeated measures ANOVA model, we analyzed the predicted number of selected attributes at the group level. As expected the predictions of the CDC model with one cutoff value did not yield a significant attractiveness effect or a difference $\times$ attractiveness interaction. In contrast, the analysis of the predictions obtained from the CDC model with two critical values yielded a significant attractiveness effect along with an attractiveness $\times$ difference interaction. This latter result supports the conjecture that the interaction is a result of an experimental manipulation which was less powerful in the high than in the low difference condition.

In conclusion, the ANOVA results and the predictions of the CDC model indicate that the majority of the subjects made use of a sequential sampling strategy taking into account the value structure of each choice pair. While the difference and the dominance effects are in agreement with the CDC model, the attractiveness effect obtained in this experiment requires a modification of the model. It is therefore important to examine the prevalence of the attractiveness effect in multiattribute choice situations. In particular, it is not clear to what extent the attractiveness effect was induced by presenting numerical values instead of descriptive features of the choice alternatives. A second experiment was therefore conducted with descriptive features to further test the CDC model and to investigate the robustness of the attractiveness effect.

\section{EXPERIMENT ॥}

The second experiment differed from the first experiment by presenting descriptive features instead of numerical ratings to the subjects. Here the task was to choose between two restaurants for a Friday evening dinner 
with a friend. The restaurants were described by 10 attributes (e.g., "cuisine," "music," "service"), and these 10 attributes had five features each. For example, the attribute "cuisine" had the five features Mexican, Italian, Chinese, Japanese, and American. During the first part of the experiment, subjects were asked to rank-order the five features of the 10 attributes. The choice situation for the subjects then consisted of two restaurants, each having one feature rank pattern. Five of these feature rank patterns are presented in Table 2. For example, if the two restaurants were assigned feature rank patterns $A$ and $B$, then for the attribute selected first by the subject, the first ranked feature was displayed for both restaurants. For the attribute selected second, the first ranked feature on that attribute was displayed for the restaurant with rank pattern $A$, and the second ranked feature was displayed for the restaurant with rank pattern B. Note that the rank pattern was realized regardless of the actual attributes selected by the subject. Also note that in rank pattern B [ 121 $\left.\begin{array}{llllll}2 & 2 & 1 & 2 & 1 & 2\end{array}\right]$, for example, the ranks of the first two attributes are repeated throughout the sequence. We employed this particular pattern of repetition to ensure that subjects arrived at a comparable evaluation of each alternative even when they differed regarding the number of inspected attributes.

\section{Method}

The experiment consisted of three parts. While subjects rank-ordered five features on each attribute during the first part, they were asked to choose between 30 pairs of alternatives during the second part of the

TABLE 2

Feature Rank Patterns of Five Sample Choice Alternatives in Experiment II

\begin{tabular}{llllll}
\hline & \multicolumn{5}{c}{ Feature rank patterns } \\
\cline { 2 - 6 } \multicolumn{1}{c}{ Attributes } & A & B & C & D & E \\
\hline Food quality & 1 & 1 & 2 & 2 & 5 \\
Music & 1 & 2 & 1 & 2 & 4 \\
Clientele & 1 & 1 & 2 & 2 & 5 \\
Specialties & 1 & 2 & 1 & 2 & 4 \\
Attire & 1 & 1 & 2 & 2 & 5 \\
Interior/decor & 1 & 2 & 1 & 2 & 4 \\
Cuisine & 1 & 1 & 2 & 2 & 5 \\
Flavor main dish & 1 & 2 & 1 & 2 & 4 \\
Service & 1 & 1 & 2 & 2 & 5 \\
Quantity & 1 & 2 & 1 & 2 & 4 \\
\hline
\end{tabular}

Note. The presentation order of the feature rank pattern of each alternative is fixed regardless of the actual attribute selected. The order of the attribute labels is determined by the subject and may therefore differ from subject to subject. 
experiment. This part was similar to the first experiment. The inspected feature pairs, the subjects' choices, and a graded preference judgment were recorded for every choice pair. During the final part of the experiment subjects were asked to both rank-order and rate the attractiveness of each feature and to give overall attractiveness judgments of the restaurants.

\section{Subjects}

Eighteen undergraduates enrolled in an introductory psychology course at the University of Illinois received credit toward a departmental requirement by participating in the experiment. Each subject was tested individually.

\section{Design and Procedure}

The choice task consisted of selecting a restaurant for a Friday evening dinner with a friend. Subjects were given 30 trials in which they had to choose between two restaurants. A $2 \times 2$ factorial combination of the attractiveness difference and attractiveness factors was used to construct the alternatives. For each cell five pairs of alternatives were generated which yielded 20 pairs of alternatives. In addition, 10 other choice pairs were mixed with the 20 choice pairs to obscure the manipulation of the alternatives' value structures. These choice pairs consisted of "mixed" alternatives; in other words, one alternative was attractive while the second alternative was either unattractive or consisted of features which received the highest and the lowest ranks. These "mixed" choice pairs were not included in the data analysis. The 20 choice pairs were constructed according to the following rules.

Attractiveness differences. Two levels of the difference factor were obtained by pairing alternatives such that the sum of their rank feature difference of the first two attributes selected differed by at most one rank unit (small difference) or by more than one rank unit (large difference). For example, an alternative with rank feature pattern $B$ was paired with an alternative with rank feature pattern $C$ in the small difference condition and an alternative with pattern $A$ with an alternative with pattern $D$ in the large difference condition (see Table 2). These differences are smaller than in the first experiment to facilitate a less restricted manipulation of the attractiveness factor.

Attractiveness of alternatives. The attractiveness of the two alternatives was manipulated by pairing two attractive alternatives or by pairing two less attractive alternatives. Attractive alternatives consisted of features that obtained the two highest rankings most of the time, while the less attractive alternatives consisted of features that obtained the two lowest rankings on the attributes most of the time. 


\section{Results}

The construction of alternatives was based on the initial rank-ordering of the features with respect to the attributes in the first part of the experiment. To examine the stability of these rank-orders, Spearman correlation coefficients were computed between the rank ordering before and after the choice part of the experiments for each of the attributes. About $80 \%$ of the correlations were equal to or higher than .90 demonstrating high but not perfect reproducibility of the rank-orders. A cross-tabulation of the first and second rank orders is displayed in Table 3 and reveals that most of the inconsistencies are a result of an interchange of adjacent rank positions. Effects due to the difference and attractiveness manipulation should be minimally affected by this interchange because most choice alternatives consisted of features ranked on either the first and second or the fourth and fifth positions.

In Fig. 5 boxplots of the number of processed attributes for the 18 subjects are presented. As in the first experiment we observed that subjects do not consider all the information available and demonstrate considerable variation when selecting the alternatives' attributes.

A repeated measures ANOVA was employed to analyze the individual and group level data. On the group level, both the difference factor $(F(1,17)=9.08, p<.01)$ and the overall attractiveness factor $(F(1,17)=$ $5.05, p<.05)$ were significant, while the interaction of both factors $(F(1,17)=0.002, p>.10)$ did not approach significance. The attractiveness and difference effect are displayed in Fig. 6. Subjects selected the largest number of attributes when the difference between undesirable alternatives was small; they selected the least number of attributes when both alternatives were attractive and the attractiveness difference between them was large. This pattern of results was observed for $72 \%$ of the subjects. Two subjects selected significantly more attributes when both alternatives were attractive.

Similar to Experiment I, a version of the CDC model was fitted to

TABLE 3

Cross-Tabulation of First (Row) and Second (Column)

RANK-ORDERING OF FEATURES

\begin{tabular}{crrrrrr}
\hline & 1 & 2 & 3 & 4 & 5 & Total \\
\hline 1 & 155 & 17 & 5 & 3 & 0 & 180 \\
2 & 18 & 135 & 20 & 6 & 1 & 180 \\
3 & 6 & 21 & 117 & 27 & 9 & 180 \\
4 & 1 & 7 & 37 & 117 & 18 & 180 \\
5 & 0 & 0 & 1 & 27 & 152 & 180 \\
Total & 180 & 180 & 180 & 180 & 180 & 900 \\
\hline
\end{tabular}




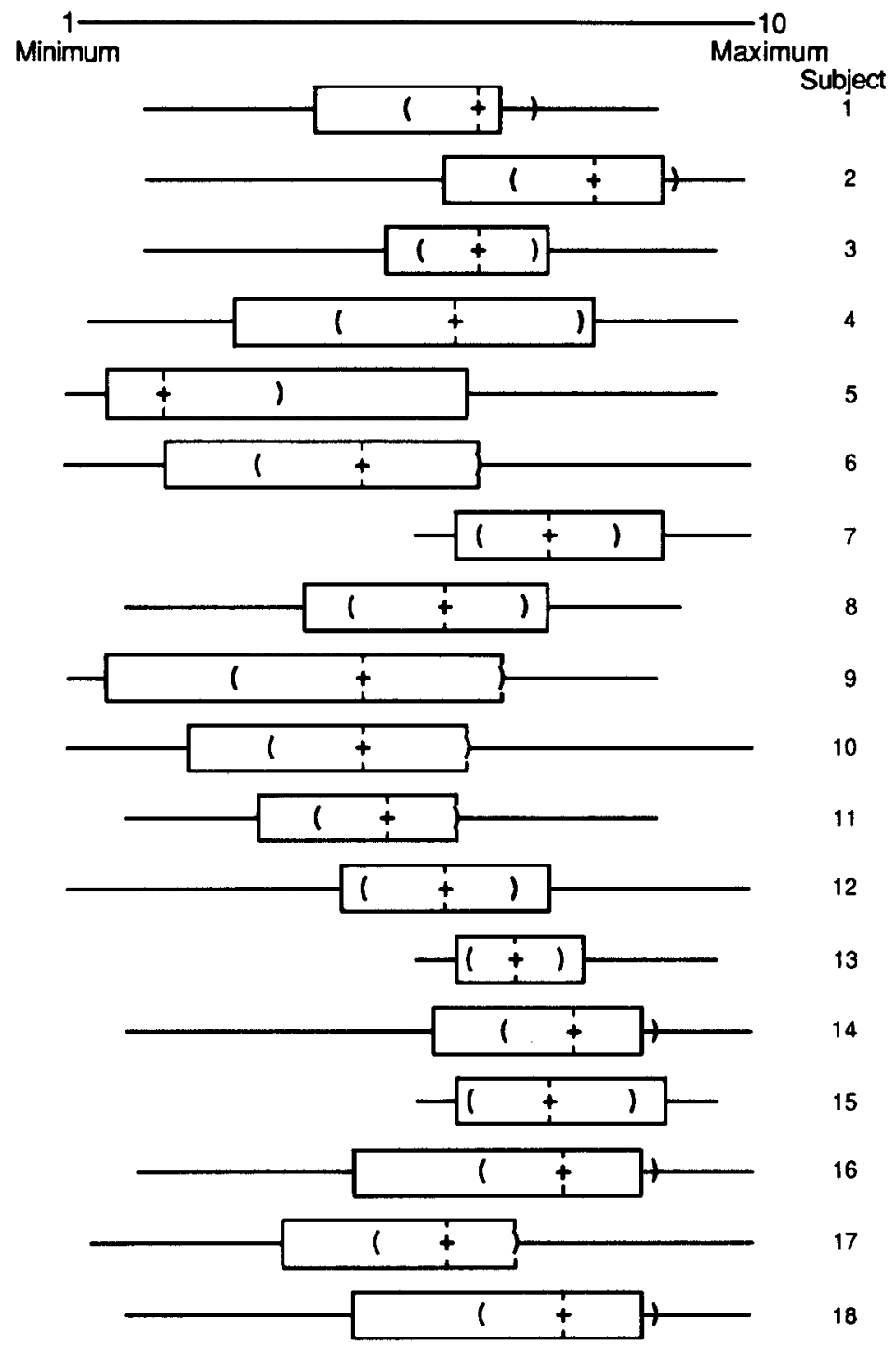

Fig. 5. Boxplots of the 18 subjects in Experiment II. (For an explanation of the boxplots, see Fig.1.)

predict the selected number of attributes. Instead of using the rankings of the features, the corresponding ratings obtained after the choice part of the experiment were used as input for the $\mathrm{CDC}$ model. These rating differences were accumulated until the sum of the rating differences exceeded a specified critical value. Using the same estimation procedure as 


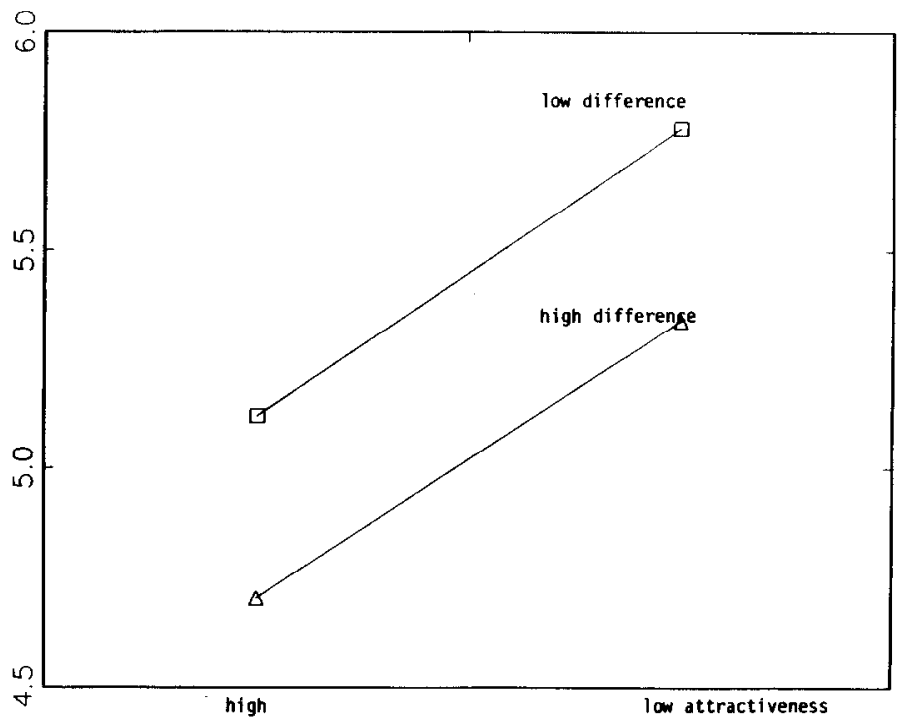

Fig. 6. The number of selected attributes in the attractiveness and difference conditions.

in the first experiment, an optimal critical value was computed for the 20 choice pairs for each subject. Due to individual differences in rating values, a sampling distribution consisting of $20 e_{P O}$ values was generated for each subject. The obtained $e_{P O}$ was compared to the 95 th percentile of the generated sampling distribution. For 14 subjects (82\%) the optimal $e_{P O}$ was larger than the 95th percentile of the sampling distribution.

When estimating a different critical value for attractive and unattractive choice alternatives, we obtained both substantially higher $e_{P O}$ 's and a significant difference between the critical values in the predicted direction. A comparison between the fitted association coefficients and their generated sampling distributions revealed that for 15 subjects $e_{P o}$ 's higher than the 95th percentile were obtained. The critical value for unattractive alternatives was significantly higher than for attractive alternatives (Wilcoxon test, $p<.001$ ).

On average the version of the CDC model with one critical value predicts $80 \%$ of the choices correctly. In comparison, the proportion of correct choice predictions is .82 if the observed number of attributes is used. Moreover, the additive (complete information processing) model predicts $80 \%$ of the choices correctly. The mean percentage of correctly predicted choices for the CDC model with different critical values for low and high attractive alternatives is $81 \%$. None of these choice predictions are, however, significantly different from each other (Wilcoxon test, $p>.10$ for all pairwise comparisons). 
Finally, a repeated measures ANOVA of the predicted number of selected attributes obtained from the CDC model with one critical value produced only a significant difference effect. The predictions of a CDC model with two critical values gave results similar to the ANOVA of the observed data by yielding a significant main effect of the difference and the attractiveness factor. Thus, as in the analysis of the first experiment, two critical values are required to capture the design effects observed in the data.

\section{Discussion}

Both the difference and attractiveness effects were replicated in the second study. It is reassuring that the presentations of numerically evaluated features and descriptive features yielded essentially the same results. While the second study was limited by the fact that only the features' rank order was manipulated, the replication of the effects provides converging evidence that ordinal information might be sufficient for studying the effect of the relationships between the alternatives. The relatively high consistency of the subjects in reproducing their rankings certainly contributed to this result.

The majority of the subjects selected more attributes when alternatives were of low attractiveness. The CDC model with one critical value cannot account for this result, while a modified version of the CDC model with two critical values is supported by both experiments. However, both versions of the $C D C$ model performed equally well in predicting the choices of the subjects. The similarity of the choice predictions is a result of the alternatives' value structures with the features' rank positions for the first and second attribute repeated for successively selected attributes. Thus, choice prediction differences between the decision models can only be observed for specifically designed value structures of the alternatives.

\section{GENERAL DISCUSSION}

Results of both studies strongly indicate subjects' selectivity and adaptiveness in their information acquisition for two different choice domains. While algebraic models based on complete information processing cannot give an explanation of these results, a sequential sampling strategy provides a good account of the data. Moreover, it is not necessary to assume that individuals adapted to changes in task and context factors by selecting different strategies (Payne et al., 1988). For example, a common inference in process tracing studies is that the amount of selected information is indicative of a compensatory versus a noncompensatory strategy (Billings \& Marcus, 1983; Ford, Schmitt, Schlechtman, Hults, \& Doherty, 1989). Our experiments show that this conclusion may not al- 
ways be accurate. A modified version of the CDC model seems entirely sufficient to explain the subjects' reactions to the variations in the value structure of the alternatives.

However, a model that is based on the assumption that subjects accumulate differences may provide only a partial description of the results. While there is little doubt that the dominance relation and the difference between two alternatives greatly affected the number of considered attributes, the attractiveness effect indicates that subjects took into account the overall attractiveness of the choice alternatives. For the same difference the majority of the subjects consistently processed less information for two attractive alternatives than for two unattractive alternatives. To account for this result, we assumed that subjects shifted their evidence criterion depending on the attractiveness level of the alternatives. This criterion shift describes the finding that the majority of subjects considered fewer attributes when the alternatives were attractive rather than unattractive. Subjects' willingness to spend more effort in selecting the better of two unattractive alternatives finds little justification from a normative perspective because the attractiveness level of an alternative does not provide any additional information over the attractiveness difference between two alternatives for making a choice. In contrast, from a costbenefit viewpoint, the attractiveness information can be useful for ensuring that indeed the better of two unattractive alternatives is chosen, while additional efforts in a choice between attractive alternatives may not seem equally rewarding because either alternative may be satisfying to the decision maker.

Further studies for testing the criterion shift hypothesis should examine the attractiveness effect under various deadline manipulations which affect the position of the evidence criterion. For example, does attractiveness play the same role when little time is available to choose among alternatives? The answer to this question should be positive if subjects are mainly interested in choosing a "satisfactory" alternative. Moreover, Busemeyer's and Townsend's (1989) decision field theory may prove to be useful in further explaining the attractiveness results by conceptualizing the choice between two attractive or between two unattractive alternatives as an approach-avoidance conflict. These authors report a series of studies which demonstrate that the deliberation time for a choice between two unattractive options is longer than between two attractive alternatives. While more research is needed to examine the attractiveness effect, it is a major obstacle for choice theories which are based on the assumption that individuals process only feature differences when comparing two alternatives.

Previous studies also obtained evidence for partial alternativewise and attributewise processing in binary choice experiments. In particular, 
Rosen and Rosenkotter (1976) found that alternativewise and attributewise information processing may occur as a function of the relationship between the attributes. They showed that independent attributes led to an attributewise processing, while dependent attributes yielded more alternativewise processing. However, dimensional processing predominated in most choice situations, and it was certainly favored by the information display used in out experiments (cf. Russo \& Dosher, 1983; Sundstrom, 1987). The results are also consistent with findings obtained by McClelland, Stewart, Judd, and Bourne (1987). They reported that subjects considered more attributes when choosing among several equally desirable alternatives than when choosing among alternatives which varied in their desirability.

The finding that information acquisition is dependent on the attractiveness of the alternatives may have other interesting implications. For example, in an unpublished pilot study conducted by one of the authors (together with Norbert Schwarz), positive and negative affect states were induced before subjects chose between two multiattribute alternatives. Consistent with the attractiveness effect, the negative affect subjects selected more information about the choice alternatives than positive affect subjects.

Finally, much decision research has been concerned with the modeling of the information integration and decision stage in choice situations. The implicit assumption of this research that information relevant to a decision is completely and costlessly available and utilized by the decision maker seems unrealistic. There is little doubt that one of the main important variables in decision making is the process of acquiring information about the choice alternatives because many, if not most decisions (especially outside the laboratory), are dependent on the outcome of this process (e.g., Connolly \& Thorn, 1987). A detailed examination of the information acquisition behavior and its integration with existing theories of choice may therefore lead to better descriptive models of real-life decision making. The experiments reported here demonstrate that the number of selected attributes is a useful dependent variable which yields reliable effects even for a small number of trials.

\section{REFERENCES}

Aschenbrenner, K. M., Albert, D., \& Schmalhofer, F. (1986). Stochastic choice heuristics. Acta Psychologica, 56, 153-166.

Aschenbrenner, K. M., Bockenholt, U., Albert, D., \& Schmalhofer, F. (1986). The selection of dimensions when choosing between multiattribute alternatives. In R. W. Scholz (Ed.), Current issues in West German decision research (pp. 63-78). Frankfurt: Lang.

Albert, D., Aschenbrenner, K. M., \& Schmalhofer, F. (1989). Cognitive choice processes and the attitude-behavior relation. In A. Upmeyer (Ed.), Attitudes and behavioral decisions (pp. 61-99). New York: Springer-Verlag. 
Batsell, R. R., \& Polking, J. (1986). A new class of market share models. Marketing Science, 4, 177-198.

Billings, R. S., \& Marcus, S. A. (1983). Measures of compensatory and noncompensatory models of decision behavior: Process tracing versus policy capturing. Organizational Behavior and Human Performance, 31, 331-352.

Birnbaum, M. H. (1974). The nonadditivity of personality impressions. Journal of Experimental Psychology Monograph, 102, 543-561.

Busemeyer, J. R. (1982). Choice behavior in a sequential decision making task. Organizational Behavior and Human Performance, 29, 175-207.

Busemeyer, J. R. (1985). Decision making under uncertainty: A comparison of simple scalability, fixed-sample, and sequential-sampling models. Journal of Experimental Psychology: Learning, Memory, and Cognition, 11, 538-564.

Busemeyer, J. R., \& Townsend, J. T. (1989). Decision field theory: A dynamic-cognitive approach to decision making. Unpublished manuscript, Purdue University.

Connolly, T., \& Thorn, B. K. (1987). Predecisional information acquisition: Effects of task variables on suboptimal search strategies. Organizational Behavior and Human Decision Processes, 39, 397-416.

Dahlstrand, U., \& Montgomery, H. (1984). Information search and evaluative processes in decision making: A computer based process tracing study. Acta Psychologica, 56, 113-123.

Ebbesen, E. B., \& Konecni, V. J. (1982). On the external validity of decision-making research: What do we know about decisions in the real world? In T. S. Wallsten (Ed.), Cognitive processes in choice and decision behavior. Hillsdale, NJ: Erlbaum.

Einhorn, H. J. (1971). The use of nonlinear, noncompensatory models as a function of task and amount of information. Organizational Behavior and Human Performance, 7, 86106.

Einhorn, H. J., Kleinmuntz, D. N., \& Kleinmuntz, B. (1979). Linear regression and process-tracing models of judgment. Psychological Review, 86, 465-485.

Ford, K. J., Schmitt, N., Schlechtman, S. L., Hults, B. M., \& Doherty, M. L. (1989). Process tracing methods: Contributions, problems, and neglected research questions. Organizational Behavior and Human Decision Processes, 43, 75-117.

Huber, $O$. (1980). The influence of some task variables on cognitive operations in an information processing decision model. Acta Psychologica, 45, 187-196.

Levin, I. P. (1975). Information integration in numerical judgments and decision processes. Journal of Experimental Psychology: General, 104, 39-53.

Lynch, J. G. (1979). Why additive utility models fail as descriptions of choice behavior. Journal of Experimental Social Psychology, 15, 397-417.

McClelland, G., Stewart, B., Judd, C., \& Bourne, L. (1987). Effects of choice task on attribute memory. Organizational Behavior and Human Decision Processes, 40, 235254.

Montgomery, H. (1977). A study of intransitive preferences using a think aloud procedure. In H. Jungermann \& G. deZeeuw (Eds.), Decision making and change in human affairs, Dordrecht: Reidel.

Montgomery, H., \& Svenson, O. (1976). On decision rules and information processing strategies for choice among multiattribute alternatives. Scandinavian Journal of Psychology, 17, 283-291.

Onken, J., Hastie, R., \& Revelle, W. (1985). Individual differences in the use of simplification strategies in a complex decision-making task. Journal of Experimental Psychology: Human Performance and Perception, 11, 14-27. 
Park, C. (1978). A seven-point scale and decision maker's simplifying choice strategy: An operationalized satisficing-plus model. Organizational Behavior and Human Performance, 21, 252-271.

Payne, J. W. (1976). Task complexity and contingent processing in decision making: An information search and protocol analysis. Organizational Behavior and Human Performance, 16, 366-387.

Payne, J. (1982). Contingent decision behavior. Psychological Bulletin, 92, 382-402.

Payne, J. W., Bettman, J. R., \& Johnson, E. J. (1988). Adaptive strategy selection in decision making. Journal of Experimental Psychology: Learning, Memory, and Cognition, 14, 534-552.

Rosen, L. D., \& Rosenkotter, P. (1976). An eye fixation analysis of choice and judgment with multiattribute stimuli. Memory \& Cognition, 4, 747-752.

Russo, J. E., \& Dosher, B. A. (1983). Strategies for multiattribute binary choice. Journal of Experimental Psychology: Learning, Memory, and Cognition, 9, 676-696.

Schmalhofer, F., Albert, D., Aschenbrenner, K. M., \& Gertzen, H. (1986). Process traces of binary choices: Evidence for selective and adaptive decision heuristics. Quarterly Journal of Experimental Psychology, 38A, 59-76.

Stine, W. W. (1989). Interobserver relational agreement. Psychological Bulletin, 106, 341347.

Sundstrom, G. A. (1987). Information search and decision making: The effects of information displays. Acta Psychologica, 65, 165-179.

Svenson, O. (1979). Process descriptions of decision making. Organizational Behavior and Human Performance, 23, 86-112.

Tversky, A. (1969). The intransitivity of preferences. Psychological Review, 76, 31-48.

Wallsten, T. S., \& Barton, C. (1982). Processing probabilistic multidimensional information for decisions. Journal of Experimental Psychology: Learning Memory, and Cognition, 8, 361-384.

Wallsten, T. S., Budescu, D., Rapoport, A., Zwick, R., \& Forsyth, B. (1986). Measuring the vague meaning of probability terms. Journal of Experimental Psychology: General, 115, 348-365.

Wright, P. (1974). The harassed decision maker: Time pressures, distractions, and the use of evidence. Journal of Applied Psychology, 59, 555-561.

Zegers, F. E. (1986). A family of chance-corrected association coefficients for metric scales. Psychometrika, 50, 17-24.

ReCEIVED: May 2, 1989 